\title{
Additive effect of tDCS combined with Peripheral Electrical Stimulation to an exercise program in pain control in knee osteoarthritis: study protocol for a randomized controlled trial
}

\author{
Cleber Luz-Santos ${ }^{1,2}$, Janine Ribeiro Camatti 1,6,7, Alaí Barbosa Paixão ${ }^{1,2}$, Katia Nunes Sá1,3, Pedro Montoya ${ }^{4}$,
} Michael Lee ${ }^{5}$ and Abrahão Fontes Baptista $1,2,6,7^{*}$

\begin{abstract}
Background: Knee osteoarthritis $(\mathrm{OA})$ has been linked to maladaptive plasticity in the brain, which may contribute to chronic pain. Neuromodulatory approaches, such as Transcranial Direct Current Stimulation (tDCS) and Peripheral Electrical Stimulation (PES), have been used therapeutically to counteract brain maladaptive plasticity. However, it is currently unclear whether these neuromodulatory techniques enhance the benefits of exercise when administered together. Therefore, this protocol aims to investigate whether the addition of tDCS combined or not with PES enhances the effects of a land-based strengthening exercise program in patients with knee OA.

Methods: Patients with knee OA $(n=80)$ will undertake a structured exercise program for five consecutive days. In addition, they will be randomized into four subgroups receiving either active anodal tDCS and sham PES (group $1 ; n=20$ ), sham tDCS and active PES (group $2, n=20$ ), sham tDCS and PES (group $3, n=20$ ), or active tDCS and PES (group 4, $n=20$ ) for $20 \mathrm{~min} /$ day for five consecutive days just prior to commencement of the exercise program. The primary outcomes will be subjective pain intensity (VAS) and related function (WOMAC). Secondary outcomes will include quality of life (SF-36), anxiety and depression symptoms (HAD), self-perception of improvement, pressure pain thresholds over the knee, quadriceps strength, and quadriceps electromyographic activity during maximum knee extension voluntary contraction. We will also investigate cortical excitability using transcranial magnetic stimulation. Outcome measures will be assessed at baseline, 1 month after, before any intervention, after 5 days of intervention, and at 1 month post exercise intervention.
\end{abstract}

Discussion: The motor cortex becomes less responsive in knee OA because of poorly adapted plastic changes, which can impede exercise therapy benefits. Adding tDCS and/or PES may help to counteract those maladaptive plastic changes and improve the benefits of exercises, and the combination of both neuromodulatory techniques must have a higher magnitude of effect. Trial registration: Brazilian Registry on Clinical Trials (ReBEC) - Effects of electrical stimulation over the skull and tight together with exercises for knee OA; protocol number RBR-9D7C7B.

Trial registration: ID: RBR-9D7C7B. Registered on 29 February 2016.

Keywords: Knee osteoarthritis, Pain, Exercise, Transcranial direct current stimulation

\footnotetext{
*Correspondence: a.baptista@ufabc.edu.br

${ }^{1}$ Functional Electrostimulation Laboratory, Health Sciences Institute, Federal

University of Bahia, Salvador, Brazil

${ }^{2}$ Graduate Program in Medicine and Health, Faculty of Medicine, Federal

University of Bahia, Salvador, Brazil

Full list of author information is available at the end of the article
} 


\section{Background}

Osteoarthritis (OA) is a chronic degenerative disease, primarily affecting the articular cartilage and subchondral bone of a synovial joint [1]. Radiographic features of OA include degradation of the articular cartilage, subchondral sclerosis and osteophyte formation [2,3]. OA primarily affects the large, weight-bearing joints such as the knee and hip $[4,5]$. The hallmark symptom of OA is chronic joint pain, which contributes to functional limitation and is a major cause of reduced quality of life. OA is the leading cause of disability affecting up to $15 \%$ of the global population, that equates to approximately 630 million people worldwide [6]. In South America, the prevalence of arthritis and rheumatism has been reported to range between 23.8 and 56.0\% [7]. In Brazil, direct OA data is not available but chronic knee pain affects approximately $11.2 \%$ of the population [8].

Knee OA affects around $10 \%$ of people over 55 years old, a quarter of whom are severely disabled [2]. Thus, knee OA poses a considerable economic burden to the community [9] due to indirect expenses incurred by patients, such as home adaptations, medications [10, 11], costs incurred for loss of employment and productivity [12]. A recent study in the United States revealed that lifetime direct medical costs of people with knee OA totaled US $\$ 129,600$ [13]. Therefore, knee OA is considered a significant global public health problem, especially with an ageing population.

The development of OA is multifactorial (Fig. 1) but some main risk factors have been identified, and these include obesity, female gender, and previous knee injury [14]. However, previous research also noted the influence of age, genetic susceptibility, trauma (acute or repetitive), muscle weakness, joint laxity, and abnormal mechanical forces, such as repetitive kneeling and squatting, as important risk factors [15].

Abnormal mechanical stresses can impede natural repair and remodeling processes of the articular cartilage $[16,17]$. The source of abnormal mechanical stress is diverse but has been associated with decreased postural control [18], muscle weakness and increased co-activation $[19,20]$, abnormal cumulative load [21, 22], joint instability [23], and the presence of abnormal tissue inside the joint (e.g., polymers of homogentisic acid oxidase in osteonecrosis) [16].

Conservative management, such as exercise therapy, designed to address issues like postural control and muscle dysfunction have limited success [24, 25]. Although the influence of $\mathrm{OA}$ on muscle strength is controversial, strengthening, stretching, and aerobic exercise are generally recommended [26]. A recent systematic review that pooled data from 44 trials concluded that land-based therapeutic exercises provide short-term pain relief (12 points/100) and improved physical function (10 points/ $100)$ for people with knee OA $[27,28]$. After around 6 months, the benefits of exercise generally plateau, and pain often persist $[27,29]$. The refractoriness to exercises needs to be addressed, and many factors may contribute.

An alternative explanation for the presence of persistent pain in OA patients is maladaptive neuroplastic changes in the spinal cord and brain [30, 31]. For example, a recent magnetic resonance imaging (MRI) study has demonstrated that patients with knee OA showed decreased volume in the somatosensory, insular, and motor cortices bilaterally. Two other clinical pain conditions were assessed including chronic back pain and complex regional pain syndrome. These changes are more pronounced in patients with a long duration of chronic pain, suggesting a positive correlation between chronicity of pain and decreased gray matter density. Similar reduction in volume has also been demonstrated in subcortical areas including the caudate nucleus and hippocampus in patients with knee $\mathrm{OA}$ and reduced volumes in those regions compromise motor control and learning [32].

The results from these imaging studies suggest that chronic pain is associated with functional changes in the brain. Studies using transcranial magnetic stimulation

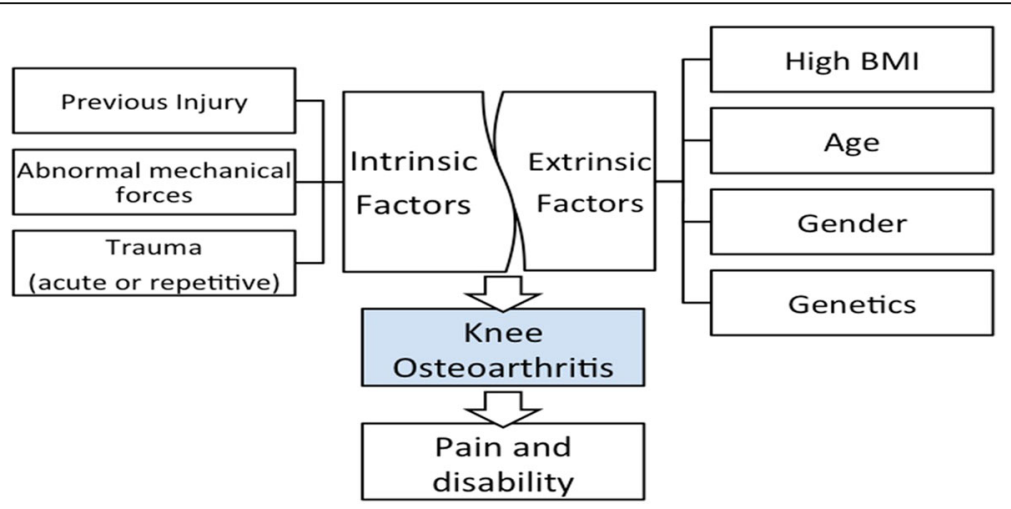

Fig. 1 Multifactorial features of knee osteoarthritis composed of intrinsic and extrinsic factors 
(TMS) have also provided useful information regarding the nature of corticomotor plasticity in chronic pain [33]. For example, several previous studies have demonstrated that various pain conditions, such as low back pain and fibromyalgia, are associated with decreased amplitude of motor evoked potentials (MEPs) and intracortical inhibition (ICI). Similar changes were also reported in studies utilizing experimental muscle pain [34-36]. To date, only few studies have examined cortical plasticity in patients with knee OA. Hunt et al. (2011) [37] in a single case study demonstrated that MEPs of the rectus femoris were decreased in unilateral knee OA, but this change could be reversed after 8 weeks of muscle strengthening, and corresponded to pain decrease. Kittelson et al. (2014) [38] found a significant negative association between the resting motor threshold (RMT) and pain, but no differences in cortical excitability measures between controls and OA subjects. Tarragó et al. (2016) demonstrated in patients with knee $\mathrm{OA}$ an association between chronicity of pain and reduction in intracortical inhibition [39]. Taken together, these findings suggest a central component related to chronic pain secondary to knee OA mostly indicating changes in M1 excitability, either decreased MEPs or motor thresholds, and decreased intracortical inhibition mechanisms and suggesting dysfunction in the interneurons which contribute to intracortical inhibitory circuits. Given the high functional connectivity of the motor cortex to other brain areas known to be involved in the pain matrix [40], such as the prefrontal cortex and thalamus [41], it might reflect not only impaired motor cortical function but also suggest a dysfunction in network connectivity as a potential mechanisms mediating the development of chronic pain [42].

As eluded to earlier, there is a growing body of evidence that many chronic pain disorders, including OA are associated with changes in motor cortical function [42-44]. Therefore, it is reasonable to suggest that treatment modalities that specifically target motor cortical function might have therapeutic value. It is well known that both exercises and electrical stimulation can induce cortical plasticity $[45,46]$. It is possible that treatment effect maybe enhanced if exercise is combined with electrical stimulation. This seems as a relevant option to reverse these changes, but they may be not sufficient to accomplish a long-term effect and interfere with maladaptive changes in the brain [47]. Associating exercises with electrical stimulation may be an option to enhance therapy efficacy. It has been shown previously that cortical excitability could be modulated both centrally using TMS and TES $[48,49]$ or peripherally using electrical stimulation [50,51], or the combination of both [52]. A novel therapeutic technique that has the potential to ameliorate maladaptive neuroplasticity in knee $\mathrm{OA}$ is
Transcranial Direct Current Stimulation (tDCS). tDCS is a simple, safe, and non-invasive technique, which involves the application of low-intensity direct electric current to the scalp [53]. tDCS is known to acutely alter excitability of the motor cortex [54-56]. Anodal stimulation of the primary motor cortex can enhance excitability by increasing the neuronal resting membrane potential, while cathodal stimulation decreases it [57]. Anodal stimulation can induce analgesic effects $[58,59]$, probably through the modulation of neuronal membrane channels resulting in local and distant plastic changes [60]. To date, tDCS has been tested in a number of pain states with low-tomoderate effect size with an averaged reduction in a Visual Analogue Score (VAS) pain score ranging between 0.18 and 0.35 [59, 61-64].

However, studies using computational modeling with a Finite Elements Model (FEM) have demonstrated low focality and dispersion of current density through the scalp during tDCS $[65,66]$ which may contribute at least in part to its reported low effect size on clinical outcomes. Using tDCS alone, without any other active intervention, may be another explanation for its low efficacy reported by previous studies [61]. One way to test the above hypotheses is to investigate to effects of combining tDCS with Peripheral Electrical Stimulation (PES). PES, similar to tDCS, PES can also transiently modulate motor cortical excitability in a bidirectional way. Sensory and nociceptive PES decrease M1 excitability, while motor PES increases M1 excitability [50]. Previous studies have investigated the effect of combining these two neuromodulatory techniques - Schabrun et al. (2013) [52] have demonstrated that when anodal tDCS is combined with motor PES (both excitatory techniques), it neutralizes the excitatory effect of each other, leading to no significant changes in M1 excitability. This phenomenon can be explained by metaplastic mechanisms (homeostatic plasticity), a phenomenon necessary to maintain physiological integrity of a biological system through the limitation extreme changes in functioning [50, 67]. However, the combination of an excitatory brain stimulation technique (anodal tDCS) with an inhibitory peripheral stimulation (sensory PES) produced a more focal modulation, as seen in Paired Associative Stimulation. In this technique, a TMS single pulse is associated with an electric pulse in the median nerve [68] to increase or decrease excitability depending on the intensity or interstimulus intervals [69, 70]. Anodal tDCS has been combined with sensory PES in chronic low back pain individuals, resulting in a more pronounced pain reduction, and M1 reorganization [71]. Taken these results together, we hypothesized that the combination of anodal tDCS with sensory PES may have additive effects to exercises in people with knee OA. 
There is encouraging evidence to suggest that the combination of tDCS and sensory PES may have the potential to reduce pain. However, it is currently unknown whether this combined approach is effective in patients with knee OA. Furthermore, it is unclear whether neuromodulation can augment the beneficial effects of exercise in patients with knee OA. Therefore, the double-blind, randomized, parallel study model was selected for this hypothesis.

\section{Hypothesis}

The addition of anodal tDCS and sensory PES to an exercise program will have a more pronounced effect on decreasing pain and improving function in people with knee OA compare to exercise alone. Specifically, our hypothesis is that this combination will promote a more robust decrease of pain magnitude and improvement in function and quality of life. We further hypothesize that these changes will be correlated with increased excitability of corticomotor and cortico-cortical connections in M1, and quadriceps strength, independent of the presence of anxiety and depression. We also hypothesize that participants submitted to this regimen will maintain the benefits of stimulation 1 month after the end of the intervention, which will be associated with a self-perception of improvement.

\section{Objectives}

\section{Primary}

The primary aim is to determine if the addition of $\mathrm{tDCS}$ and PES to an exercise program is more effective in reducing pain, improving function and quality of life in patients with chronic knee OA compared to exercise alone.

\section{Secondary}

To assess the effect of combining tDCS, PES and exercise, on motor threshold, cortical excitability, and cortical silent period of the rectus femoris, vastus medialis, and vastus lateralis muscles to exercise alone.

To correlate the neurophysiological findings with parameters of pain, muscle strength, global function related to OA, anxiety and depression symptoms, quality of life, and treatment perception from subjects.

\section{Methods}

\section{Target population and sample}

Participants will be recruited from public announcements and interviewed at the Laboratory of Functional Electrical Stimulation, Federal University of Bahia, Salvador, Bahia, Brazil. Sample size was estimated based on an effect size of 0.50 on pain relief measured by VAS score, study power of $80 \%$, and four groups, with a total of 15 subjects per group. Total sample size was increased to 20 to account for loss to follow-up.

\section{Inclusion criteria}

Participants will be included in this study if they have OA of the knee based on the clinical and radiological criteria defined by the American College of Rheumatology Society. These criteria include:

1. Age older than 50 years, knee pain on most days of the past month, osteophytes on plain X-ray and pain or difficulty in rising from sitting or climbing stairs [72]

2. Have a Chronic Pain Grade (CPG) score equal to or greater than II $[73,74]$

\section{Exclusion criteria}

Participants will be excluded if they:

1. Have a contraindication for TMS use such as: existence of metal in the skull or implanted devices, epilepsy history, pregnancy, use of drugs that might affect cortical electrical activity (anticonvulsants, antidepressants or antipsychotics), and complications with exposure to magnetic fields (TMS or MRI)

2. Have a disease history that might interfere with the knee OA, becoming a confounding bias fibromyalgia, systemic lupus erythematosus, fractures of the knee region, knee prosthesis, low back impairment - that cause symptoms in the knee and peripheral nerves

3. Are incapable of comprehend the content from the assessments tools

\section{Discontinuity criteria}

Procedures will be discontinued if:

- Moderate-to-severe adverse events are present, even related to electrical stimulation, exercises, or electrophysiological assessment

- Participants who initiate any other medical intervention to treat knee OA or other that may interfere with the results of this study

Subjects who initiate any of the treatments described below will be discontinued from the study:

- Physiotherapeutic treatment (conventional physiotherapy, postural re-education, pilates)

- Psychiatric treatment (associated anxiety and depression)

- Inclusion of new drug treatment for pain due to knee OA

- Treatment for weight reduction for obese individuals (aerobic exercise program)

- Fitness muscle training 


\section{Study design}

Study participants will be assessed on four different occasions: (1) baseline assessment (day 1) 1 month before the commencement of intervention; (2) reassessment a month later (day 30), (3) participants will be submitted to an intervention consisting of electrical stimulation applied before the structured exercise program for five consecutive days, (d) reassessment at the completion of the exercise program (day 36) and 1 month after the last day of intervention (day 66) (Fig. 2).

An experienced physiotherapist blinded to the study will perform initial neurophysiological assessment. The subjects will be randomly allocated by the research manager, which will only be involved in this study phase, at one of the four groups through a lottery system generated from a random-number table (www.randomization.com). Allocation sequence implementation mechanism will be by opaque and sealed envelopes. Access to this envelope will be exclusive to the physiotherapist who will apply the intervention techniques. The absence of blinding will not be allowed in this study. In the event of a breach of the blinding, the participant will be removed from the research protocol. The SPIRIT checklist with the registration events, interventions, evaluations and visits for participants can be verified in Fig. 3.

The same physiotherapist that performed the baseline assessment will teach and supervise the exercise program, common to all groups. Another researcher will be responsible for administering electrical stimulation interventions ( $\mathrm{tDCS}, \mathrm{PES}$, and their combinations) and will not be involved in any other component of the study. The allocation concealment will be evaluated at the end of the intervention by custom questionnaire. The study participants will complete an adverse effect questionnaire at the end of the trial.

\section{Research groups}

All groups will receive a supervised exercise program:
1. Active anodal tDCS associated with sham PES $(n=20)$

2. Sham tDCS associated with active PES $(n=20)$

3. Sham tDCS associated with sham PES $(n=20)$

4. Active anodal tDCS associated with active PES $(n=20)$

\section{Strategies to improve adhesion to the intervention}

All the participants will receive compensations for transportation to and from the testing laboratory as well as a meal allowance. At the end of the study the intervention that promoted greater reduction in pain will be offered to those who did not receive it. To minimize withdrawal of participants during the study, a collaborating researcher will make telephone contact to confirm the appointment. Despite this, if the participant is absent, they may attend within $48 \mathrm{~h}$ for the evaluations without risk of exclusion from the protocol.

\section{Intervention description \\ Electrical stimulation}

For the electrical stimulation protocol, participants will be comfortably seated on a chair at the Functional Electrical Stimulation Laboratory in the Health Science Institute of Federal University of Bahia. The procedures will be clearly explained and subjects will be encouraged to ask questions regarding the experimental procedures. The tDCS protocol will consist of a direct current stimulation lasting 20 $\mathrm{min}$, of 2-mA amplitude, for five consecutive days before the exercise protocol, through a proper stimulator device (Soterix, New York, NY, USA). The anode will be placed on the primary motor cortex (M1 - C3 or C4, per the 10/ 20 international electroencephalogram (EEG) system) contralateral to the painful knee or the most symptomatic one in case of bilateral pain. The cathode will be placed on the opposite supraorbital region (Fp1 or Fp2, per 10/20 international EEG system). For the sham tDCS group the electrodes will be placed and the electric current connected for $30 \mathrm{~s}$, then turned off and removed at the end of the 20-min period.

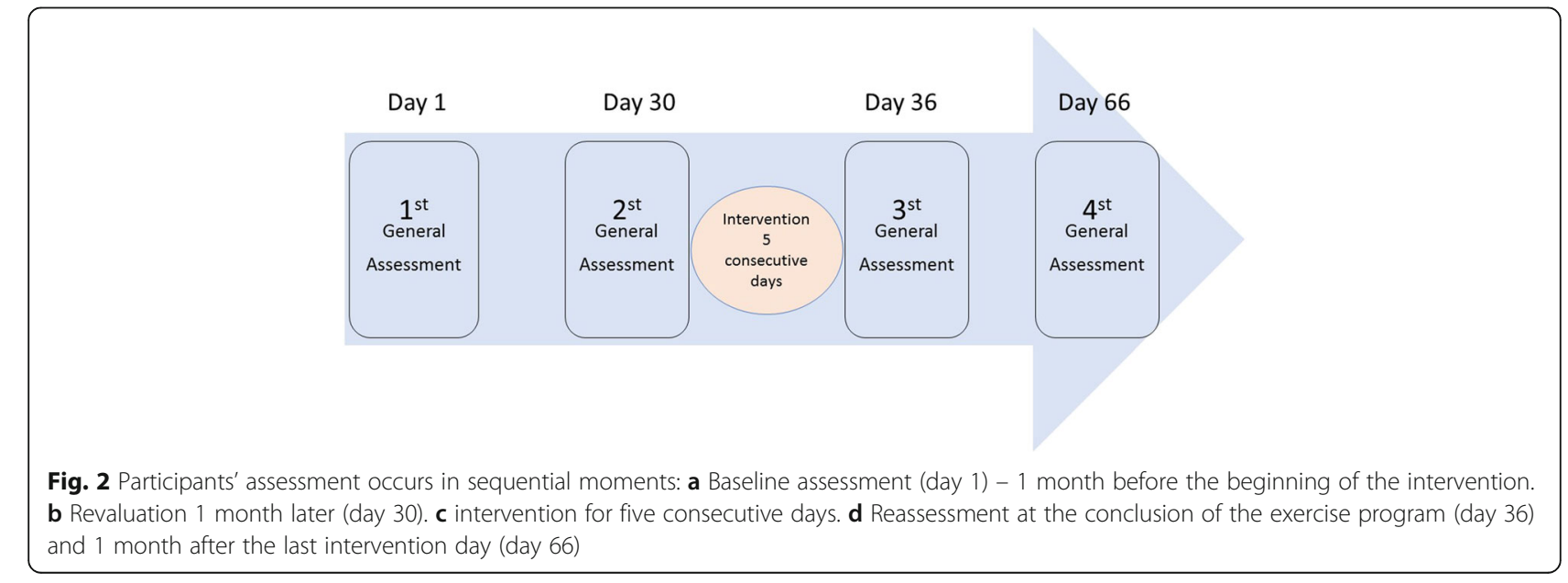




\begin{tabular}{|c|c|c|c|c|c|c|}
\hline \multirow[b]{3}{*}{ TIMEPOINT** } & \multicolumn{6}{|c|}{ STUDY PERIOD } \\
\hline & \multirow{2}{*}{$\begin{array}{c}\text { Enrolment } \\
-t_{1} \\
\text { Day }-8 \\
\end{array}$} & \multirow{2}{*}{$\begin{array}{c}\text { Allocation } \\
0 \\
\text { Day } 1 \text { (BASELINE) }\end{array}$} & \multicolumn{3}{|c|}{ Post-allocation } & \multirow{2}{*}{$\begin{array}{c}\text { Close-out } \\
T_{3} \\
\text { Day } 66\end{array}$} \\
\hline & & & $\begin{array}{c}\boldsymbol{t}_{1} \\
\text { Day } 30\end{array}$ & $\begin{array}{c}\text { Intervention } \\
\text { (Days 31-35) }\end{array}$ & $\begin{array}{c}t_{2} \\
\text { Day } 36\end{array}$ & \\
\hline \multirow{5}{*}{$\begin{array}{r}\text { ENROLMENT: } \\
\text { Eligibility screen and verbal consent } \\
\text { Contraindications tms } \\
\text { Informed consent } \\
\text { Allocation }\end{array}$} & & & & & & \\
\hline & $\mathrm{X}$ & & & & & \\
\hline & $\mathrm{X}$ & & & & & \\
\hline & $\mathrm{X}$ & & & & & \\
\hline & & $\mathrm{X}$ & & & & \\
\hline \multirow{2}{*}{\multicolumn{7}{|c|}{$\begin{array}{l}\text { INTERVENTIONS: } \\
\text { Active anodal tDCS associated with sham }\end{array}$}} \\
\hline & & & & & & \\
\hline \multicolumn{7}{|l|}{ Sham tDCS associated with active PES } \\
\hline \multirow{2}{*}{\multicolumn{7}{|c|}{$\begin{array}{l}\text { Sham tDCS associated with sham PES } \\
\text { Active anodal tDCS associated with active }\end{array}$}} \\
\hline & & & & & & \\
\hline \multicolumn{7}{|l|}{ ASSESSMENTS: } \\
\hline Outcome: Questionnaire & & $\mathrm{X}$ & $\mathrm{X}$ & & $\mathrm{X}$ & $\mathrm{X}$ \\
\hline WOMAC & & $\mathrm{X}$ & $\mathrm{X}$ & & $\mathrm{X}$ & $\mathrm{X}$ \\
\hline$H A D$ & & $\mathrm{X}$ & $\mathrm{X}$ & & $\mathrm{X}$ & $\mathrm{X}$ \\
\hline$S F-36$ & & $\mathrm{X}$ & $\mathrm{X}$ & & $\mathrm{X}$ & $\mathrm{X}$ \\
\hline Adverse events & & $\mathrm{X}$ & $\mathrm{X}$ & & $\mathrm{X}$ & $\mathrm{X}$ \\
\hline \multicolumn{7}{|l|}{ Outcome: Physical measures } \\
\hline EVA & & $\mathrm{X}$ & $\mathrm{X}$ & & $\mathrm{X}$ & $\mathrm{X}$ \\
\hline Pressure Pain Thresholds & & $\mathrm{X}$ & $\mathrm{X}$ & & $\mathrm{X}$ & $\mathrm{X}$ \\
\hline \multirow{2}{*}{$\begin{array}{r}\text { Eletrctromyography } \\
\text { Outcome: Electrophysiological } \\
\text { measures }\end{array}$} & & $\mathrm{X}$ & $\mathrm{x}$ & & $\mathrm{X}$ & $\mathrm{X}$ \\
\hline & & $x$ & $x$ & & $x$ & $x$ \\
\hline TMS & & $\mathrm{X}$ & $\mathrm{X}$ & & $\mathrm{X}$ & $\mathrm{X}$ \\
\hline EEG & & $\mathrm{X}$ & $X$ & & $\mathrm{X}$ & $\mathrm{X}$ \\
\hline
\end{tabular}

Fig. 3 Schematic diagram of enrollment, interventions, assessments, and visits for participants

PES of the knee joint will be performed on its lateral and medial portions with a clinical pulse generator (Endophasys, KLD Medical Products, Brazil). Only the symptomatic side will be stimulated or the more symptomatic (in case of bilateral symptoms). A biphasic, symmetric current will be used for stimulation with a 0.25ms pulse width, frequency of $10 \mathrm{~Hz}$, with an amplitude at the sensory threshold, lasting $20 \mathrm{~min}$, with the subject sitting comfortably at rest. Two surface electrodes of silicon-carbon bond, $5 \times 5 \mathrm{~cm}$, fixed on skin by Velcro will be used at each side of the joint. For sham stimulation the same electrode montage will be used with active stimulation on a sensory threshold for $30 \mathrm{~s}$ and then returned to zero. Electrodes will be removed after 20 min. tDCS and PES will start and stop at the same time during each session.

\section{Exercise}

The exercises will be based on a previously described protocol (Bennel and Dobson (2014) [75]), and will be taught by a trained physiotherapist at the first day of intervention. Assistance to proper performance and progression will happen during the next 4 days' intervention. In summary, the protocol consists on strengthening exercises for muscles of the knees and hips. The intervention will be carried out with three series of 8 to 12 repetitions (Additional file 1).

\section{Outcomes}

\section{Primary outcome measures}

Pain

The intensity of the symptomatic knee (or the knee with greatest symptoms if both knees are affected) will be assessed before and after each intervention session. In case of equal magnitude of pain on both knees, the dominant side will be evaluated. Pain magnitude will be assessed at all meetings with the participants, through a 0-10 VAS. When this meeting includes the intervention, pain will be assessed before and after the procedures. In general evaluations, the pressure pain threshold on the medial and lateral sides of both knees will be assessed using a digital pressure pain threshold (EMG System, São José dos Campos, Brazil). Pressure pain threshold at the medial and lateral sides of both knees will be assessed using a digital pressure pain threshold meter (EMG System, São José dos Campos, Brazil) on days 1, 30, 36, and 66. The mean of three measures at each point will be used for analysis. Together, pain, stiffness and physical function will be assessed through the Brazilian version of the Western Ontario and McMaster Universities Osteoarthritis Index (WOMAC) [76].

\section{Secondary outcomes}

The secondary outcomes will be assessed at each of the four general assessment sessions (days 1, 30, 36, and 66). Quality of life will be assessed through the 36-item Short 
Form (SF-36) questionnaire [77] and symptoms of depression and anxiety through the Hospital Anxiety and Depression Scale (HADS) [78].

Quadriceps muscle strength will be assessed through a digital dynamometer (EMG System, Brazil) positioned at a right angle to the ankle. The subject will be seated with hip and knees at $90^{\circ}$. The make test paradigm will be used. Three maximal isometric contractions of both knees will be recorded, each lasting $6 \mathrm{~s}$, with 1 min rest between them. Standard verbal encouragement will be provided to all participants to generate maximal force. The average of the three recordings will be used for subsequent analysis.

Cortical excitability will be assessed using a Magstim BiStim stimulator (Magstim Co. Ltd, Dyfed, UK). The quadriceps area corresponding to the painful knee and the anterior superior iliac spine (ASIS) will be cleaned with alcohol. Self-adhesive electromyography (EMG) electrodes $\mathrm{Ag} / \mathrm{AgCl}$ (Noraxon, Scottsdale, AZ, USA) will be positioned on the muscle belly of the rectus femoris, vastus lateralis and vastus medialis muscles. The RMS EMG activity will be pre-amplified x3000, filtered at $1-2000 \mathrm{~Hz}$, and sampled at $4000 \mathrm{~Hz}$ using a 1401 acquisition system and Signal v.06 software (Cambridge Electronic Design, Cambridge, UK). During the testing procedure the subject should extend the knee until an EMG activity of $100 \mu \mathrm{V}$ is reached. The participant will be asked to maintain this level of contraction during the entire evaluation period.

The vertex will be marked at the intersection of the interaural and nasion to inion lines according to the 10/ 20 EEG system. The subject will be comfortably seated on a proper chair and kept awake during the entire evaluation protocol. A polyester cap previously marked with a $1 \times 1$ $\mathrm{cm}$ grid will be positioned on the subject's head and will serve as reference for the TMS. The TMS will be applied by the examiner using a figure-of-eight coil to deliver single and paired pulsed TMS to the motor cortex, while RMS EMG activity will be monitored in real time through Signal v.06 software (Cambridge Electronic Design, Cambridge, UK). The spot where a balanced EMG activity is found for the rectus femoris, vastus lateralis, and vastus medialis activation will be considered as the "hot spot." Active motor threshold (AMT) will be estimated as the minimum stimulator intensity that evokes responses in the target muscle of at least $200 \mu \mathrm{V}$ of peak-to-peak amplitude. AMT will be estimated through computer software (Motor Threshold Assessment Tool, www.clinicalre searcher.org). MEP amplitude will be estimated by the mean of 10 pulses at $120 \%$ of AMT. For short-interval intracortical inhibition (SICI) and intracortical facilitation (ICF), a subthreshold conditioning pulse (80\% AMT) will be delivered followed by a suprathreshold test stimulus (120\% AMT) separated by pre-defined intervals of $2 \mathrm{~ms}$ for SICI and $15 \mathrm{~ms}$ for ICF.

\section{Potential TMS risks}

Potential risks were described by Rossi et al. (2009) and Lefaucheur et al. (2011) [79, 80]. All potential risks are listed and summarized on the subject's Informed Consent Form. Subjects will complete an adverse effects questionnaire for TMS and tDCS just after each intervention.

\section{Ethical aspects}

The protocol meets the legal requirements involving research in humans, according to the Declaration of Helsinki. All participants will be briefed on the objectives and will sign the Consent Form. This protocol was approved by the Ethics Committee of the Health Sciences Institute of the Federal University of Bahia under number 1,378,100, and in the Brazilian System of Clinical Trials Registration (REBEC) under number RBR-9D7C7B. Possible changes to the protocol will be communicated to the Ethics Committee, REBEC, and participants. The findings will be forwarded to the participants and collaborators through a simplified report in an accessible language. Documents will not be made public without the permission of the participant and the resulting publications will have no identification of the subjects. The data will be archived with the principal investigator for 5 years and then destroyed. Researchers are physiotherapists who are able to help in cases of research-related harms.

The authors of the publications will be considered the researchers who meet the following criteria:

1. Substantially contributing to the design and planning, or acquisition of data, or analysis and interpretation of data

2. Participate in article writing or critical intellectual review

3. Review and approve the final version to be published

The researchers declare no conflicts of interest with financial agents and access to data will be restricted to key researchers.

\section{Consent to publish}

Written consent will be obtained from the participants for the publication of their individual data and accompanying images in this manuscript. The Consent Form will be archived by the researcher in charge and will be available for review by Editor-in-Chief. The images provided to illustrate the exercise program were granted by one of the collaborators through the term of assignment of the image use.

\section{Data analysis}

All statistical procedures will be performed according to the principles of intention-to-treat. A linear mixed model will be used to identify the differences in pain intensity 
(VAS), PPT, and WOMAC scores between the groups with real stimulation (tDCS, PES and tDCS + PES) and sham interventions across the periods of assessment (factors intervention and time). Anxiety and depression will be analyzed as covariables. When necessary, post hoc comparisons will be performed using the Bonferroni correction for multiple comparisons. Analysis of the cumulative proportion of respondents with different cutoff points will be employed to analyze clinical analgesic response between groups according to Farrar et al. (2006) [81]. Correlation measurements will be performed between the clinical variables (VAS, PPT, and WOMAC scores and secondary variables (quality of life and TMS variables PEM, IIC and FIC). All data will be analyzed using software SPSS v.23.0. Statistical significance will be set as $p<0.05$.

\section{Discussion}

We adhere to the recommendations of SPIRIT (Standard Protocol: Recommendations for Interventional Testing) in the development and reporting of our protocol (Additional file 2). The exercises have demonstrated positive effects on pain and function in various chronic pain conditions [82]. The results obtained can be optimized when associated with non-invasive neuromodulatory techniques. The procedures with the most evidence are from tDCS [83-85] and PES [86, 87]. The combination with the largest effect size is not sufficiently elucidated in the scientific literature and ideal estimation parameters of the modalities are controversial. The present study used the parameters with the highest scientific evidence for the proposed clinical outcomes.

Exercise combined with neuromodulatory techniques may reverberate in the motor cortex [46, 70]. This fact suggests that clinical and electrophysiological variables are positively correlate. Reorganization of the quadriceps motor cortex after an exercise program supports this correlation [70].

Clinical impacts may represent significant changes in the cortical activity of the subject with knee OA. The understanding of the relationship between brain and muscle may lead the physician to propose interventions that amplify this connection and significantly improve function.

\section{Benefits}

All individuals will receive specific follow-up from physiotherapists, who will guide the implementation and evolution of therapeutic exercises with a good level of evidence for the treatment of OA of the knee. Additionally we believe that the electrical resources tested can increase the effects of the exercises. If these resources prove to be effective, the best therapy will be offered to those who did not receive it.

\section{Trials status}

Estimated enrollment is 15 participants. The study start date was June 2016, and the estimated study completion date is June 2018.

\section{Additional files}

\section{Additional file 1: Description of the exercise program with images,} progressions and repetitions. (PDF $1335 \mathrm{~kb}$ )

Additional file 2: SPIRIT 2013 Checklist: recommended items to address in a clinical trial protocol and related documents*. (DOCX $49 \mathrm{~kb}$ )

\section{Authors' contributions \\ $C S$ is the principal investigator of the study. $A B$ is the study coordinator. $C S, A B$, $J C, M L, P M$ and AP participated actively in the methodological development and writing of the manuscript. KS, ML, PM reviewed and approved the final manuscript and assisted in statistical planning. CS and AP are physiotherapists and will perform the evaluations and interventions in the subjects of the research. All authors read and approved the final manuscript.}

\section{Ethics approval and consent to participate}

The current test protocol was approved by the Research Ethics Committee (Institute of Health Sciences, Federal University of Bahia) with number $1,378,100$. The identity of the participants will be treated with professional standards of confidentiality, in accordance with Brazilian legislation (Resolution 466/12 of the National Health Council), using the information only for academic and scientific purposes. All participants will provide informed written consent prior to participating in the trial.

Consent for publication

Not applicable.

\section{Competing interests}

The authors declare that they have no competing interests regarding this study.

\section{Author details}

${ }^{1}$ Functional Electrostimulation Laboratory, Health Sciences Institute, Federal University of Bahia, Salvador, Brazil. ${ }^{2}$ Graduate Program in Medicine and Health, Faculty of Medicine, Federal University of Bahia, Salvador, Brazil. ${ }^{3}$ Bahian School of Medicine and Public Health, Salvador, Brazil. ${ }^{4}$ Research Institute on Health Sciences, University of Balearic Islands, Palma de Majorca, Spain. ${ }^{5}$ Graduate School of Health, Discipline of Physiotherapy, University of Technology Sydney, Sydney, NSW, Australia. ${ }^{6}$ Center for Mathematics, Computation and Cognition, Federal University of ABC, São Bernardo do Campo, São Paulo 09.080-045, Brazil. ${ }^{7}$ Graduate Program in Neuroscience and Cognition, Federal University of ABC, São Bernardo do Campo, Brazil.

Received: 10 April 2017 Accepted: 8 November 2017

Published online: 21 December 2017

\section{References}

1. Felson DT, Gross KD, Nevitt MC, Yang M, Lane NE, Torner JC, et al. The effects of impaired joint position sense on the development and progression of pain and structural damage in knee osteoarthritis. Arthritis Rheum. 2009;61:1070-6. [Internet]. Available from: http://www.ncbi.nlm.nih. gov/pubmed/19644911. Accessed 5 Dec 2016

2. Jordan KM, Arden NK, Doherty M, Bannwarth B, Bijlsma JWJ, Dieppe P, et al. EULAR Recommendations 2003: an evidence based approach to the management of knee osteoarthritis: Report of a Task Force of the Standing Committee for International Clinical Studies Including Therapeutic Trials (ESCISIT). Ann Rheum Dis. 2003;62:1145-55. [Internet]. Available from: http://www.ncbi.nlm.nih.gov/pubmed/14644851. Accessed 5 Dec 2016.

3. Peat G, McCarney R, Croft P. Knee pain and osteoarthritis in older adults: a review of community burden and current use of primary health care. Ann Rheum Dis. 2001;60:91-7. [Internet]. Available from: http://www.ncbi.n/m.nih. gov/pubmed/11156538. Accessed 5 Dec 2016. 
4. O'Reilly SC, Jones A, Muir KR, Doherty M. Quadriceps weakness in knee osteoarthritis: the effect on pain and disability. Ann Rheum Dis. 1998;57: 588-94. [Internet]. Available from: http://www.ncbi.nlm.nih.gov/pubmed/ 9893569.Accessed 5 Dec 2016.

5. Osiri M, Wongchinsri J, Ukritchon S, Hanvivadhanakul P, Kasitanon N, Siripaitoon B. Comprehensibility, reliability, validity, and responsiveness of the Thai version of the Health Assessment Questionnaire in Thai patients with rheumatoid arthritis. Arthritis Res Ther. 2009;11:R129. [Internet]. Available from: http://www.ncbi.nlm.nih.gov/pubmed/19709443. Accessed 5 Dec 2016.

6. Udgata S. Osteoarthritis: an overview. IOSR J Pharm Biol Sci Ver. 2014;9: 2319-7676.

7. Wong R, Davis AM, Badley E, Grewal R, Mohammed M, Davis A. Prevalence of arthritis and rheumatic diseases around the world. A growing burden and implications for health care needs models of care in arthritis, Bone \& Joint Disease (MOCA). 2010.

8. Sa KN, Pereira C de M, Souza RC, Baptista AF, Lessa I. Knee pain prevalence and associated factors in a Brazilian population study. Pain Med. 2011;12: 394-402. [Internet] Available from: https://www.ncbi.nlm.nih.gov/pubmed/ 19488666. Accessed 5 Dec 2016.

9. Neogi T, Zhang Y. Epidemiology of OA. Rheum Dis Clin North Am. 2013;39:1-19.

10. MacLean $\mathrm{CH}$, Knight $\mathrm{K}$, Paulus $\mathrm{H}$, Brook RH, Shekelle PG. Costs attributable to osteoarthritis. J Rheumatol. 1998;25:2213-8. [Internet]. Available from: http://www.ncbi.nlm.nih.gov/pubmed/9818666. Accessed 16 Dec 2015.

11. Maetzel A. Der Gebrauch von Nutzwerten im gesundheitsökonomischen Vergleich von Interventionen bei verschiedenen Krankheitsbildern. Zeitschrift für Rheumatol. 2004;63:380-4. Available from: http://www.ncbi. nlm.nih.gov/pubmed/15517298.

12. Gupta S, Hawker GA, Laporte A, Croxford R, Coyte PC. The economic burden of disabling hip and knee osteoarthritis $(\mathrm{OA})$ from the perspective of individuals living with this condition. Rheumatol. 2005;44:1531-7. [Internet] Available from: http://www.ncbi.nlm.nih.gov/pubmed/16091394. Accessed 16 Dec 2015

13. Losina E, Paltiel AD, Weinstein AM, Yelin E, Hunter DJ, Chen SP, et al. Lifetime medical costs of knee osteoarthritis management in the United States: impact of extending indications for total knee arthroplasty. Arthritis Care Res. 2015;67:203-15.

14. Silverwood V, Blagojevic-Bucknall M, Jinks C, Jordan JL, Protheroe J, Jordan $\mathrm{KP}$, et al. Current evidence on risk factors for knee osteoarthritis in older adults: a systematic review and meta-analysis. Osteoarthritis Cartilage. 2015; 23:507-15. Elsevier.

15. Heidari B. Knee osteoarthritis prevalence, risk factors, pathogenesis and features: Part I. Casp J Intern Med. 2011;2:205-12. Babol University of Medical Sciences.

16. Brandt KD, Dieppe P, Radin E. Etiopathogenesis of osteoarthritis. Med Clin North Am. 2009;93:24,xv. [Internet]. Available from: http://www.ncbi.nlm.nih. gov/pubmed/19059018. Accessed 18 Dec 2014.

17. Hunter DJ. Imaging insights on the epidemiology and pathophysiology of osteoarthritis. Rheum Dis Clin North Am. 2009;35:447-63. [Internet]. Available from: http://www.ncbi.nlm.nih.gov/pubmed/19931798. Accessed 5 Dec 2016.

18. Sanchez-Ramirez DC, van der Leeden M, Knol DL, van der Esch M, Roorda LD, Verschueren S, et al. Association of postural control with muscle strength, proprioception, self-reported knee instability and activity limitations in patients with knee osteoarthritis. J Rehabil Med. 2013;45:192-7. [Internet]. Available from: https://www.ncbi.nlm.nih.gov/pubmed/23223845. Accessed 5 Dec 2016.

19. Hubley-Kozey CL, Hill NA, Rutherford DJ, Dunbar MJ, Stanish WD. Coactivation differences in lower limb muscles between asymptomatic controls and those with varying degrees of knee osteoarthritis during walking. Clin Biomech. 2009;24:407-14. [Internet] Available from: http:// www.ncbi.nlm.nih.gov/pubmed/19303179. Accessed 16 Dec 2015.

20. Knoop J, van der Leeden M, van der Esch M, Thorstensson CA, Gerritsen M, Voorneman RE, et al. Association of lower muscle strength with self-reported knee instability in osteoarthritis of the knee: results from the Amsterdam osteoarthritis cohort. Arthritis Care Res. 2012;64:38-45. [Internet] Available from: https:/www.ncbi.nlm.nih.gov/pubmed/22213723. Accessed 5 Dec 2016.

21. Block JA, Shakoor N. Lower limb osteoarthritis: biomechanical alterations and implications for therapy. Curr Opin Rheumatol. 2010;22:544-50. [Internet]. Available from: http://www.ncbi.nlm.nih.gov/pubmed/20592605. Accessed 5 Dec 2016

22. Maly MR. Abnormal and cumulative loading in knee osteoarthritis. Curr Opin Rheumatol. 2008;20:547-52. [Internet] Available from: https://www. ncbi.nlm.nih.gov/pubmed/18698176. Accessed 5 Dec 2016.
23. van der Esch M, Knoop J, van der Leeden M, Voorneman R, Gerritsen M Reiding D, et al. Self-reported knee instability and activity limitations in patients with knee osteoarthritis: results of the Amsterdam osteoarthritis cohort. Clin Rheumatol. 2012;31:1505-10. [Internet]. Available from: http:// www.ncbi.nlm.nih.gov/pubmed/22729472. Accessed 5 Dec 2016.

24. Mat S, Tan MP, Kamaruzzaman SB, Ng CT. Physical therapies for improving balance and reducing falls risk in osteoarthritis of the knee: a systematic review. Age Ageing. 2015;44:16-24. [Internet]. Available from: http://www. ncbi.nlm.nih.gov/pubmed/25149678. Accessed 3 Dec 2015.

25. Iwamoto J, Sato Y, Takeda T, Matsumoto H. Effectiveness of exercise for osteoarthritis of the knee: a review of the literature. World J Orthop. 2011;2: 37-42. [Internet] Available from: https://www.ncbi.n/m.nih.gov/pubmed/ 22474634. Accessed 17 Dec 2015.

26. Jansen MJ, Viechtbauer W, Lenssen AF, Hendriks EJM, de Bie RA. Strength training alone, exercise therapy alone, and exercise therapy with passive manual mobilisation each reduce pain and disability in people with knee osteoarthritis: a systematic review. J Physiother. 2011;57:11-20. [Internet]. Available from: http://www.ncbi.nlm.nih.gov/pubmed/21402325. Accessed 6 Dec 2016.

27. Fransen M, McConnell S, Harmer AR, Van der Esch M, Simic M, Bennell KL. Exercise for osteoarthritis of the knee: a Cochrane systematic review. $\mathrm{Br}$ J Sports Med. 2015;49:1554-7.

28. Fransen M, McConnell S. Exercise for osteoarthritis of the knee. Cochrane Database Syst Rev. 2008;1:CD004376. [Internet]. Available from: http://www.ncbi. nlm.nih.gov/pubmed/18843657. Accessed 1 Dec 2014. v. 2015 Jan 9;1:CD004376.

29. Tanaka R, Ozawa J, Kito N, Moriyama H. Efficacy of strengthening or aerobic exercise on pain relief in people with knee osteoarthritis: a systematic review and meta-analysis of randomized controlled trials. Clin Rehabil. 2013;27:1059-71.

30. Schaible H-G. Mechanisms of chronic pain in osteoarthritis. Curr Rheumatol Rep. 2012;14:549-56. [Internet]. Available from: https://www.ncbi.nlm.nih. gov/pubmed/22798062. Accessed 5 Dec 2016.

31. Howard MA, Sanders D, Krause K, O'Muircheartaigh J, Fotopoulou A, Zelaya $F$, et al. Alterations in resting-state regional cerebral blood flow demonstrate ongoing pain in osteoarthritis: An arterial spin-labeled magnetic resonance imaging study. Arthritis Rheum. 2012;64:3936-46. [Internet]. Available from: https://www.ncbi.n/m.nih.gov/pubmed/22933378. Accessed 5 Dec 2016.

32. Mao CP, Bai ZL, Zhang XN, Zhang QJ, Zhang L. Abnormal subcortical brain morphology in patients with knee osteoarthritis: a cross-sectional study. Front Aging Neurosci. 2016;8:3. Frontiers Media SA.

33. Hallett M. Transcranial magnetic stimulation: a primer. Neuron. 2007;55:18799. [Internet]. Available from: https://www.ncbi.n/m.nih.gov/pubmed/ 17640522. Accessed 10 Jul 2014.

34. Schabrun SM, Hodges PW. Muscle pain differentially modulates short interval intracortical inhibition and intracortical facilitation in primary motor cortex. J Pain. 2012;13:187-94. [Internet]. Available from: https://www.ncbi. nlm.nih.gov/pubmed/22227117. Accessed 7 Feb 2017.

35. Tsao H, Galea MP, Hodges PW. Reorganization of the motor cortex is associated with postural control deficits in recurrent low back pain. Brain. 2008;131:2161-71. [Internet]. Available from: https://www.ncbi.nlm.nih.gov/ pubmed/18669505. Accessed 7 Feb 2017.

36. Baudic S, Attal N, Mhalla A, CiampideAndrade D, Perrot S, Bouhassira D. Unilateral repetitive transcranial magnetic stimulation of the motor cortex does not affect cognition in patients with fibromyalgia. J Psychiatr Res. 2013:47:72-7. [Internet]. Available from: https://www.ncbi.nlm.nih.gov/ pubmed/23079535. Accessed 5 Dec 2016

37. Hunt MA, Zabukovec JR, Peters S, Pollock CL, Linsdell MA, Boyd LA. Reduced quadriceps motor-evoked potentials in an individual with unilateral knee osteoarthritis: a case report. Case Rep Rheumatol. 2011;2011:537420. [Internet]. Available from: https://www.ncbi.nlm.nih.gov/pubmed/22937446. Accessed 7 Feb 2017.

38. Kittelson AJ, Thomas AC, Kluger BM, Stevens-Lapsley JE. Corticospinal and intracortical excitability of the quadriceps in patients with knee osteoarthritis. Exp Brain Res. 2014;232:3991-9. [Internet]. Available from: https://www.ncbi.nlm.nih.gov/pubmed/25183161. Accessed 23 Dec 2015.

39. Tarragó Mda G1, Deitos A, Brietzke AP, Vercelino R, Torres IL, Fregni F Caumo W. Descending control of nociceptive processing in knee osteoarthritis is associated with intracortical disinhibition: an exploratory study. Medicine (Baltimore). 2016;95:e3353. [Internet]. Available from: https:// www.ncbi.nlm.nih.gov/pubmed/27124022. Accessed 5 Dec 2016.

40. Fomberstein K, Qadri S, Ramani R. Functional MRI and pain. Curr Opin Anaesthesiol. 2013;26:588-93. [Internet]. Available from: http://www.ncbi. nlm.nih.gov/pubmed/23995063. Accessed 21 Feb 2017. 
41. Wilcox CE, Mayer AR, Teshiba TM, Ling J, Smith BW, Wilcox GL, et al. The subjective experience of pain: an FMRI study of percept-related models and functional connectivity. Pain Med. 2015;16:2121-33. [Internet]. Available from: http://www.ncbi.nlm.nih.gov/pubmed/25989475. Accessed 21 Feb 2017.

42. Parker RS, Lewis GN, Rice DA, McNair PJ. Is motor cortical excitability altered in people with chronic pain? A systematic review and meta-analysis. Brain Stimul. 2016;9:488-500. [Internet]. Available from: https://www.ncbi.nlm.nih. gov/pubmed/27133804. Accessed 7 Feb 2017.

43. DosSantos MF, Ferreira N, Toback RL, Carvalho AC, DaSilva AF. Potential Mechanisms Supporting the Value of Motor Cortex Stimulation to Treat Chronic PainSyndromes. Front Neurosci. 2016;10:18.

44. Mhalla A, de Andrade DC, Baudic S, Perrot S, Bouhassira D. Alteration of cortical excitability in patients with fibromyalgia. Pain. 2010;149:495-500. [Internet]. Available from: http://www.ncbi.nlm.nih.gov/pubmed/20356675. Accessed 22 Feb 2015

45. Ostadan F, Centeno C, Daloze J-F, Frenn M, Lundbye-Jensen J, Roig M. Changes in corticospinal excitability during consolidation predict acute exercise-induced off-line gains in procedural memory. Neurobiol Learn Mem. 2016;136:196-203. [Internet]. Available from: http://www.ncbi.nlm.nih. gov/pubmed/27773595. Accessed 21 Feb 2017.

46. Stavrinos EL, Coxon JP. High-intensity Interval Exercise Promotes Motor Cortex Disinhibition and Early Motor SkillConsolidation. J Cogn Neurosci. 2017;29(4):593-604

47. Shanahan CJ, Hodges PW, Wrigley TV, Bennell KL, Farrell MJ. Organisation of the motor cortex differs between people with and without knee osteoarthritis. 2011.

48. Medeiros LF, de Souza ICC, Vidor LP, de Souza A, Deitos A, Volz MS, et al. Neurobiological effects of transcranial direct current stimulation: a review. Front Psychiatry. 2012;3:110. [Internet]. Available from: https://www.ncbi.nlm. nih.gov/pubmed/23293607. Accessed 28 Nov 2015

49. Inukai Y, Saito K, Sasaki R, Tsuiki S, Miyaguchi S, Kojima S, et al. Comparison of three non-invasive transcranial electrical stimulation methods for increasing cortical excitability. Front Hum Neurosci. 2016;10:668. [Internet]. Available from: http://www.ncbi.nlm.nih.gov/pubmed/28082887. Accessed 21 Feb 2017.

50. Chipchase LS, Schabrun SM, Hodges PW. Peripheral electrical stimulation to induce cortical plasticity: a systematic review of stimulus parameters. Clin Neurophysiol. 2011;122:456-63. [Internet]. Available from: https://www.ncbi. nlm.nih.gov/pubmed/20739217. Accessed 6 Dec 2016.

51. Chipchase LS, Schabrun SM, Hodges PW. Corticospinal excitability is dependent on the parameters of peripheral electric stimulation: a preliminary study. Arch Phys Med Rehabil. 2011;92:1423-30. [Internet]. Available from: http://www.ncbi.nlm.nih.gov/pubmed/21620374. Accessed 6 Dec 2016.

52. Schabrun SM, Chipchase LS, Zipf N, Thickbroom GW, Hodges PW. Interaction between simultaneously applied neuromodulatory interventions in humans. Brain Stimul. 2013;6:624-30. [Internet]. Available from: http:// www.ncbi.nlm.nih.gov/pubmed/23088854. Accessed 12 Jun 2015.

53. Schabrun SM, Chipchase LS. Priming the brain to learn: the future of therapy? Man Ther. 2012;17:184-6. [Internet]. Available from: http://www. ncbi.nlm.nih.gov/pubmed/22197081. Accessed 6 Dec 2016.

54. Romero Lauro L, Rosanova M, Mattavelli G, Convento S, Pisoni A, Opitz A, et al. TDCS increases cortical excitability: direct evidence from TMS-EEG. Cortex. 2014;58:99-111.

55. Nitsche MA, Liebetanz D, Tergau F, Paulus W. Modulation of cortical excitability by transcranial direct current stimulation. Nervenarzt. 2002;73:332-5.

56. Alonzo A, Brassil J, Taylor JL, Martin D, Loo CK, Lang N, et al. Daily transcranial direct current stimulation (tDCS) leads to greater increases in cortical excitability than second daily transcranial direct current stimulation. Brain Stimul. 2012:5:208-13.

57. Di Lazzaro V, Ranieri F, Profice P, Pilato F, Mazzone P, Capone F, et al. Transcranial direct current stimulation effects on the excitability of corticospinal axons of the human cerebral cortex. Brain Stimul. 2013;6:641-3. [Internet]. Available from: https://www.ncbi.nlm.nih.gov/pubmed/23085442. Accessed 6 Dec 2016.

58. Marlow NM, Bonilha HS, Short EB. Efficacy of transcranial direct current stimulation and repetitive transcranial magnetic stimulation for treating fibromyalgia syndrome: a systematic review. Pain Pr. 2013;13:131-45. [Internet]. Available from: http:/www.ncbi.nlm.nih.gov/pubmed/22631436. Accessed 16 Dec 2015.

59. O'Connell NE, Cossar J, Marston L, Wand BM, Bunce D, De Souza LH, et al. Transcranial direct current stimulation of the motor cortex in the treatment of chronic nonspecific low back pain: a randomized, double-blind exploratory study. Clin J Pain. 2013;29:26-34. [Internet]. Available from: https://www.ncbi.nlm.nih.gov/pubmed/23221623. Accessed 6 Dec 2016.
60. Vaseghi B, Zoghi M, Jaberzadeh S. How does anodal transcranial direct current stimulation of the pain neuromatrix affect brain excitability and pain perception? A randomised, double-blind, sham-control study. PLoS One. 2015;10:e0118340.

61. O'Connell NE, Wand BM, Marston L, Spencer S, Desouza LH. Non-invasive brain stimulation techniques for chronic pain. Cochrane Database Syst Rev. 2014;(4)

62. Fregni F, Boggio PS, Lima MC, Ferreira MJL, Wagner T, Rigonatti SP, et al. A sham-controlled, phase II trial of transcranial direct current stimulation for the treatment of central pain in traumatic spinal cord injury. Pain. 2006;122: 197-209. [Internet]. Available from: https://www.ncbi.nlm.nih.gov/pubmed/ 16564618. Accessed 6 Dec 2016.

63. Fregni F, Gimenes R, Valle AC, Ferreira MJL, Rocha RR, Natalle L, et al. A randomized, sham-controlled, proof of principle study of transcranial direct current stimulation for the treatment of pain in fibromyalgia. Arthritis Rheum. 2006;54:3988-98. [Internet]. vailable from: https://www.ncbi.nlm.nih. gov/pubmed/17133529. Accessed 6 Dec 2016

64. Valle A, Roizenblatt S, Botte S, Zaghi S, Riberto M, Tufik S, et al. Efficacy of anodal transcranial direct current stimulation (tDCS) for the treatment of fibromyalgia: results of a randomized, sham-controlled longitudinal clinical trial. J Pain Manag. 2009;2:353-61. [Internet]. Available from: http://www. ncbi.n/m.nih.gov/pubmed/21170277. Accessed 6 Dec 2016.

65. Sadleir RJ, Vannorsdall TD, Schretlen DJ, Gordon B. Transcranial direct current stimulation (tDCS) in a realistic head model. Neuroimage. 2010:51:1310-8.

66. Miranda PC, Lomarev M, Hallett M. Modeling the current distribution during transcranial direct current stimulation. Clin Neurophysiol. 2006;117:1623-9.

67. Park SH, Hwangbo G. Effects of combined application of progressive resistance training and Russian electrical stimulation on quadriceps femoris muscle strength in elderly women with knee osteoarthritis. J Phys Ther Sci Japan. 2015;27:729-31

68. Carson RG, Kennedy NC. Modulation of human corticospinal excitability by paired associative stimulation. Front Hum Neurosci. 2013;7:823. [Internet]. Available from: https://www.ncbi.nlm.nih.gov/pubmed/24348369. Accessed 6 Dec 2016

69. Andrews RK, Schabrun SM, Ridding MC, Galea MP, Hodges PW, Chipchase LS. The effect of electrical stimulation on corticospinal excitability is dependent on application duration: a same subject pre-post test design. J Neuroeng Rehabil. 2013;10:51. [Internet]. Available from: https://www.ncbi. nlm.nih.gov/pubmed/23758902. Accessed 6 Dec 2016.

70. Ridding MC, Brouwer B, Miles TS, Pitcher JB, Thompson PD. Changes in muscle responses to stimulation of the motor cortex induced by peripheral nerve stimulation in human subjects. Exp Brain Res. 2000;131:135-43. [Internet]. Available from: http://www.ncbi.nlm.nih.gov/pubmed/10759179. Accessed 19 Aug 2015.

71. Schabrun SM, Jones E, Elgueta Cancino EL, Hodges PW. Targeting chronic recurrent low back pain from the top-down and the bottom-up: a combined transcranial direct current stimulation and peripheral electrical stimulation intervention. Brain Stimul. 2014:7:451-9.

72. Menkes CJ. Radiographic criteria for classification of osteoarthritis. J Rheumatol Suppl. 1991;27:13-5. [Internet]. Available from: http://www.ncbi. nlm.nih.gov/pubmed/2027113. Accessed 21 Feb 2017.

73. Thomas E, Dunn KM, Mallen C, Peat G. A prognostic approach to defining chronic pain: application to knee pain in older adultsł. Pain. 2008;139:38997. [Internet]. Available from: http://www.ncbi.nlm.nih.gov/pubmed/ 18583051. Accessed 21 Feb 2017.

74. Elliott AM, Smith BH, Smith WC, Chambers WA. Changes in chronic pain severity over time: the Chronic Pain Grade as a valid measure. Pain. 2000;88: 303-8. [Internet]. Available from: http://www.ncbi.nlm.nih.gov/pubmed/ 11068118. Accessed 21 Feb 2017.

75. Bennell KL, Dobson F, Hinman RS. Exercise in osteoarthritis: moving from prescription to adherence. Best Pract Res Clin Rheumatol. 2014;28:93-117. [Internet]. Available from: http://www.ncbi.nlm.nih.gov/pubmed/24792947. Accessed 7 Apr 2017.

76. Bellamy N, Buchanan WW, Goldsmith CH, Campbell J, Stitt LW. Validation study of WOMAC: a health status instrument for measuring clinically important patient relevant outcomes to antirheumatic drug therapy in patients with osteoarthritis of the hip or knee. J Rheumatol. 1988;15:183340. [Internet]. Available from: http://www.ncbi.nlm.nih.gov/pubmed/ 3068365. Accessed 18 Dec 2014 
77. Ciconelli RM, Ferraz MB, Santos W, Meinão I, Quaresma MR. BrazilianPortuguese version of the SF-36. A reliable and valid quality of life outcome measure Rev. bras. reumatol. 1999; 39(3):143-50. Available from: http://bases. bireme.br/cgi-bin/wxislind.exe/iah/online/?lsisScript=iah/iah.xis\&src= google\&base $=$ LILACS\&lang $=$ p\&nextAction $=$ Ink\&exprSearch $=$ 296502\&indexSearch=ID.

78. Pais-Ribeiro J, Silva I, Ferreira T, Martins A, Meneses R, Baltar M. Validation study of a Portuguese version of the Hospital Anxiety and Depression Scale. Psychol Health Med. 2007;12:225-35-7. [Internet]. Available from: http:// www.tandfonline.com/doi/abs/10.1080/13548500500524088. Accessed 7 Apr 2017.

79. Lefaucheur J-P, André-Obadia N, Poulet E, Devanne H, Haffen E, Londero A, et al. Recommandations françaises sur l'utilisation de la stimulation magnétique transcrânienne répétitive (rTMS): règles de sécurité et indications thérapeutiques. Neurophysiol Clin Neurophysiol. 2011:41:221-95. [Internet]. Available from: http://www.ncbi.nlm.nih.gov/pubmed/22153574. Accessed 7 Apr 2017.

80. Rossi S, Hallett M, Rossini PM, Pascual-Leone A, Safety of TMS Consensus Group. Safety, ethical considerations, and application guidelines for the use of transcranial magnetic stimulation in clinical practice and research. Clin Neurophysiol. 2009;120:2008-39. [Internet]. Available from: http://www.ncbi. nlm.nih.gov/pubmed/19833552. Accessed 7 Apr 2017.

81. Farrar JT. Chapter 56 The measurement and analysis of pain symptoms. Handb Clin Neurol. 2006;81:833-42.

82. Ambrose KR, Golightly YM. Physical exercise as non-pharmacological treatment of chronic pain: why and when. Best Pract Res Clin Rheumatol. 2015;29:120-30. [Internet]. Available from: http://www.ncbi.nlm.nih.gov/ pubmed/26267006. Accessed 4 Oct 2016.

83. Chang W-J, Bennell KL, Hodges PW, Hinman RS, Liston MB, Schabrun SM. Combined exercise and transcranial direct current stimulation intervention for knee osteoarthritis: protocol for a pilot randomised controlled trial. BM Open. 2015;5:e008482. [Internet]. Available from: https://www.ncbi.nlm.nih. gov/pubmed/26297371. Accessed 24 Dec 2015.

84. de AC DN, Grecco LAC, Galli M, Fregni F, Oliveira CS. Effect of transcranial direct-current stimulation combined with treadmill training on balance and functional performance in children with cerebral palsy: a double-blind randomized controlled trial. PLoS One. 2014;9:e105777. [Internet]. Available from: https://www.ncbi.nlm.nih.gov/pubmed/25171216. Accessed 24 Dec 2015.

85. Park SD, Kim JY, Song HS. Effect of application of transcranial direct current stimulation during task-related training on gait ability of patients with stroke. J Phys Ther Sci. 2015;27:623-5. [Internet]. Available from: https:// www.ncbi.nlm.nih.gov/pubmed/25931694. Accessed 24 Dec 2015.

86. Elboim-Gabyzon M, Rozen N, Laufer Y. Does neuromuscular electrical stimulation enhance the effectiveness of an exercise programme in subjects with knee osteoarthritis? A randomized controlled trial. Clin Rehabil. 2013; 27:246-57. [Internet]. Available from: https://www.ncbi.nlm.nih.gov/ pubmed/22952305. Accessed 23 Nov 2015.

87. Gruet M, Temesi J, Rupp T, Levy P, Verges S, Millet GY. Dynamics of corticospinal changes during and after high-intensity quadriceps exercise. Exp Physiol. 2014;99:1053-64. [Internet]. Available from: http://www.ncbi. nlm.nih.gov/pubmed/24907029. Accessed 7 Apr 2017.

\section{Submit your next manuscript to BioMed Central and we will help you at every step:}

- We accept pre-submission inquiries

- Our selector tool helps you to find the most relevant journal

- We provide round the clock customer support

- Convenient online submission

- Thorough peer review

- Inclusion in PubMed and all major indexing services

- Maximum visibility for your research

Submit your manuscript at www.biomedcentral.com/submit
Biomed Central 